\title{
Atendimentos de emergência a vítimas de violências e acidentes: diferenças no perfil epidemiológico entre o setor público e o privado. VIVA - Campinas/SP, 2009
}

\author{
Emergency care for victims of violence and accidents: \\ differences in the epidemiological profile between the public \\ and private health services. VIVA - Campinas, São Paulo, Brazil, 2009
}

Ana Paula Belon ${ }^{1}$

Naoko Yanagizawa Jardim da Silveira ${ }^{2}$

Marilisa Berti de Azevedo Barros ${ }^{1}$

Caroline Baldo ${ }^{1}$

Marta Maria Alves da Silva ${ }^{3}$

${ }^{1}$ Departamento de Saúde Coletiva, Faculdade de Ciências Médicas,

Universidade Estadual de Campinas. Rua Tessália

Vieira de Camargo 126, CCAS/Depto de Saúde

Coletiva, Cid. Universitária

Zeferino Vaz. 13083-887

Campinas SP.

paulabelon@gmail.com

${ }^{2}$ Coordenadoria de

Vigilância em Saúde,

Secretaria Municipal de

Saúde de Campinas.

${ }^{3}$ Coordenação Geral de

Vigilância de Doenças e

Agravos Não

Transmissíveis, Secretaria

de Vigilância em Saúde,

Ministério da Saúde.

\begin{abstract}
The scope of this study is to analyze the differences in the profile of emergency care for external causes between public and private emergency departments. With data come from VIVACampinas 2009, the association between the nature of healthcare and the characteristics of the victims was verified using the chi-square test. Using Poisson regression, proportion ratios of care in the public and private network were estimated. In the sample of 1094 victims, 67.8\% were treated by public health. Traffic accidents, animal-related accidents, and assaults were 2 times higher in public units, whereas collisions with objects and sprains were $75 \%$ and 2.7 times higher in private units. Cranium-encephalic trauma/polytrauma and cuts/lacerations were 3.8 times and $61 \%$ more frequent in public care, while victims with no injuries, with dislocations/sprains or fractures being predominant in private care. Head and multiple organ injuries, road accident and work-related injuries, the use of public transport or mobile emergency care services/ambulances were predominant in public care. Revealing significant differences in care in public and private care can contribute to the organization of healthcare.
\end{abstract}

Key words Emergency medical services, Accidents, Violence, Public sector, Private sector
Resumo O objetivo deste estudo foi analisar as diferenças no perfil dos atendimentos de emergência por causas externas, entre as unidades de saúde públicas/conveniadas ao SUS e as privadas. Com dados do VIVA-Campinas 2009, foi verificada a associação entre natureza do serviço de saúde e características das vítimas, evento e atendimento usando teste qui-quadrado. A partir da regressão de Poisson, foram estimadas as razões entre a proporção de atendimentos da rede pública e da privada. O setor público respondeu por $67,8 \%$ dos atendimentos na amostra de 1094 vítimas. Acidentes de transportes, acidentes com animais $e$ agressões foram 2 vezes mais frequentes nas unidades públicas; já choques contra objeto e entorses foram $75 \%$ e 2,7 vezes superiores nas privadas. Traumatismos crânio-encefálicos/politraumatismos e cortes/lacerações foram 3,8 vezes e $61 \%$ mais frequentes no setor público, enquanto ocorrências sem lesão física, com luxações/entorses ou fraturas predominaram no privado. Vítimas com lesões na cabeça e em múltiplos órgãos, ocorrências em vias públicas, eventos relacionados ao trabalho, uso de transportes coletivos e SAMU/resgate/ambulâncias prevaleceram na rede pública. O estudo, ao apontar significativas diferenças entre os eventos atendidos na rede pública e privada, pode contribuir na organização da assistência à saúde.

Palavras-chave Serviços médicos de emergência, Acidentes, Violência, Setor público, Setor privado, Estudos transversais 


\section{Introdução}

Violências e acidentes constituem grave problema de saúde pública no Brasil com enorme impacto econômico e social nos sistemas de saúde e previdenciário e no aparato da segurança pública, além de causar severos danos à saúde e à qualidade de vida da população. As causas externas, que englobam as violências e os acidentes, constituem a terceira causa de mortalidade na população geral e a sexta de internações no país ${ }^{1}$. De acordo com estimativas da Organização PanAmericana de Saúde, a taxa de mortalidade por causas externas no Brasil, para o período de 2003 a 2005, foi de 84,3 óbitos por 100 mil habitantes valor elevado em comparação a países como Canadá $(35,9)$, Estados Unidos $(49,7)$, México $(52,9)$ e Chile $(28,9)^{2}$. E sua tendência, no país, tem sido ascendente com aumento de 3,9\% entre 2000 e $2009^{3}$. Analisando por causas específicas, esta variação foi ainda maior, sendo de $352,5 \%$ para as quedas no mesmo nível e 224,2\% para acidentes com motocicletas ${ }^{3}$.

Assim como outros municípios brasileiros, Campinas, no estado de São Paulo, presenciou um significativo aumento das taxas de mortalidade por violência e acidentes na década de noventa, o que causou a redução da expectativa de vida masculina em 1 ano entre 1991 e $2000^{4}$. Embora tenha havido declínio significativo das causas externas, particularmente graças aos homicídios, a partir do ano de 2000, produzindo um aumento de 2,3 anos na expectativa de vida dos homens entre 2000 e $2005^{4}$, as taxas do município ainda permanecem elevadas, especialmente entre adultos jovens ${ }^{5}$. Especificamente sobre a tendência da acidentalidade no município, estudo recente aponta que a proporção de ocorrência de acidentes de trânsito com vítimas e os coeficientes de mortalidade entre ocupantes de veículos e pedestres apresentaram ligeiro aumento entre 2000 e 2008, bem como indica que $49,3 \%$ do total de vítimas de acidentes fatais eram ocupantes de motocicletas ${ }^{6}$.

Como os acidentes e as violências resultam em mortalidade prematura e evitável, impõem incapacidades às vítimas e geram altos custos coletivos, a ocorrência destes eventos exige constante monitoramento e vigilância para subsidiar a avaliação e o aperfeiçoamento das políticas públicas vigentes.

No Brasil, a maioria das investigações sobre causas externas apoia-se em fontes oficiais de registro contínuo, usando dados do Sistema de Informações sobre Mortalidade (SIM) e do Sistema de Informações Hospitalares (SIH). Contudo, estas fontes não captam as lesões de menor gravidade que são as responsáveis pela grande demanda nos serviços de urgência e emergência.

Visando coletar informações sobre morbidade dos eventos que não necessariamente resultam em internações ou óbitos, e considerando que as unidades de emergência são a porta de entrada para o tratamento das lesões ocasionadas por acidentes e violências, o Ministério da Saúde implantou, em 2006, o Sistema de Vigilância de Violências e Acidentes (VIVA) que prevê a realização periódica de inquéritos em serviços de urgência e emergência das capitais brasileiras e de alguns outros municípios ${ }^{7,8}$.

O município de Campinas, no estado de São Paulo, tem participado do projeto VIVA desde o seu início, tendo conduzido inquéritos nos anos de 2006, 2007, 2009 e 2011. O levantamento VIVA de Campinas de 2009 apresentou um caráter inovador, sendo o único município que, em sua amostra, teve incorporados os atendimentos realizados no setor privado, além dos que ocorrem no setor público, como usualmente é feito nos inquéritos VIVA. Devido à expressiva importância da assistência suplementar à saúde em Campinas com $55 \%$ da população com planos de saúde privados ${ }^{9}$ e à existência de uma extensa rede privada de hospitais com pronto-atendimentos e pronto-socorros para atender a demanda dos pacientes com planos de saúde privados, foi considerado adequado que a pesquisa incluísse, neste município, uma amostra geral dos atendimentos de violências e acidentes, abrangendo todas as unidades de urgência e emergência públicas ou credenciadas ao SUS e os serviços privados de saúde que respondem por grande parcela dos atendimentos de emergência do setor.

Portanto, o inquérito VIVA de Campinas em 2009 viabiliza a identificação das possíveis diferenças entre os perfis das vítimas de violências e acidentes que procuram os serviços do SUS e o segmento que busca atendimento no setor privado. Esta análise pode contribuir para melhor entendimento dos resultados das pesquisas realizadas pelo VIVA, usualmente desenvolvidas apenas nos serviços públicos, como também detectar as diferenças das demandas no setor público e privado e identificar as características e a gravidade dos eventos atendidos em cada esfera, fornecendo subsídios para estratégias de organização da assistência à saúde. O objetivo deste estudo foi, portanto, analisar as diferenças no perfil dos atendimentos de emergência a vítimas de violências e acidentes entre as unidades de saúde públicas ou conveniadas ao SUS e as da rede privada no município de Campinas em 2009. 


\section{Métodos}

Trata-se de um estudo de corte transversal que utilizou os dados coletados pelo inquérito do Sistema de Vigilância de Violências e Acidentes (VIVA) conduzido em Campinas no ano de 2009.

Neste município, a rede de atenção ao paciente vítima de violências e acidentes é composta pela rede pública e privada de hospitais e de unidades de pronto-atendimentos. A rede instalada de atenção pública ao paciente é descentralizada e hierarquizada, composta por 2 hospitais universitários (Hospital das Clínicas da Unicamp e Hospital e Maternidade Dr. Celso Pierro) e o Hospital Municipal Dr. Mário Gatti, os quais são considerados referência para traumas. Além destes, compõem a rede pública cinco unidades de Pronto-atendimento regionalizadas. A rede privada conta com vários hospitais com pronto-atendimento ou pronto-socorro para atender a demanda dos pacientes com planos de saúde privados. O município dispõe ainda de serviço de atendimento móvel, como o SAMU, que faz a avaliação da gravidade do caso e o encaminhamento dos pacientes para serviços públicos ou privados. Outras unidades de pré-atendimento são o resgate e as ambulâncias particulares ou de concessionárias de rodovias.

O estudo incluiu os atendimentos realizados no mês de outubro em 15 serviços de urgência e emergência do município, dos quais oito públicos ou credenciados ao SUS (três unidades de referência e cinco unidades de pronto-atendimento) e sete do setor privado, que abrangem as principais unidades de saúde voltadas ao atendimento às causas externas.

Foi definido um tamanho mínimo de amostra de 750 entrevistas de forma a garantir a estimativa de uma proporção (entre 5 a $50 \%$ ) com um coeficiente de variação inferior a $30 \%$ e erro padrão menor que $3^{7}$. Para cumprir com esta meta e garantir a representatividade dos atendimentos em cada unidade de saúde, e também considerando os resultados dos inquéritos prévios em 2006 e 2007 e os dados do Sistema de Informações Hospitalares do SUS, foi realizado um sorteio de 6 turnos de 12 horas (diurnos ou noturnos) em cada um dos 15 serviços de saúde incluídos na amostra, totalizando 90 turnos sorteados. A unidade primária de amostragem foi constituída pelo turno de 12 horas. O processo de sorteio foi a amostragem por conglomerado em um estágio. Todos os pacientes, vítimas de acidentes e violências, que demandavam a unidade durante o turno sorteado deveriam ser entrevistados. Assim, a população do estudo foi com- posta por todas as vítimas de acidentes e violências atendidas pelos serviços de urgência e emergência do município durante o mês de outubro de 2009.

$\mathrm{O}$ instrumento de coleta de dados foi o formulário padronizado para todo o país, contendo questões fechadas sobre o perfil da pessoa atendida, as características do evento e do atendimento, e incluindo ainda dados da lesão e da evolução do caso nas primeiras 24 horas. Os pacientes ou seus responsáveis eram abordados diretamente por entrevistadores previamente treinados, que dispunham de um manual do entrevistador e eram supervisionados.

As variáveis analisadas neste estudo foram: a natureza jurídica da unidade de saúde (público/ conveniado ou privado), dados referentes ao perfil sociodemográfico da vítima (sexo, faixa etária, raça/cor da pele, escolaridade), características do evento (tipo de ocorrência, local do evento, natureza da lesão corporal, parte do corpo atingida, se a ocorrência estava relacionada ao trabalho, suspeita e uso de bebidas alcóolicas) e características do atendimento (meio de locomoção para chegar ao serviço de saúde, período e dia de atendimento e evolução do caso nas primeiras 24 horas).

Os dados foram digitados em banco previamente elaborado pelo Ministério da Saúde e os dados coletados foram submetidos a análises de consistência. As análises estatísticas foram realizadas com o uso do programa Stata 9.2 (Stata Corp., College Station, Estados Unidos). Foram estimadas as proporções com intervalos de confiança de 95\% (IC 95\%) das características dos atendimentos. A associação entre as diversas variáveis e a natureza jurídica das unidades de saúde foi verificada usando o teste qui-quadrado com nível de significância de 5\%. Por meio da regressão de Poisson, foram calculadas razões entre as proporções (RP) de atendimentos realizados no setor público e no privado e os respectivos intervalos de 95\% de confiança (IC 95\%).

O termo de consentimento livre e esclarecido foi substituído pelo consentimento verbal da vítima ou do seu acompanhante, uma vez que se trata de um inquérito específico de vigilância epidemiológica conduzido em escala nacional ${ }^{7}$.

\section{Resultados}

Foram realizadas 1224 entrevistas no inquérito VIVA de Campinas de 2009. Dos 90 plantões sorteados, foi possível realizar a pesquisa em 88 . Após análises de consistência e verificação de duplicidade de fichas, foram excluídos 130 for- 
mulários, gerando uma perda de 10,6\%. Desse modo, foram analisados os dados de 1094 entrevistas, das quais 742 foram conduzidas em serviços de saúde públicos ou conveniados e 352 no setor privado. Os primeiros responderam por $67,8 \%$ (IC 95\% 65,0-70,6) dos atendimentos a urgência e emergência do município e cerca de $88,4 \%$ das ocorrências $(n=1.090)$ eram de residentes do município de Campinas.

Quanto ao perfil da vítima, 59,7\% eram do sexo masculino, $38,1 \%$ tinham entre 20 e 39 anos de idade, $63,7 \%$ se autodeclararam brancas e $38,2 \%$ informaram ter concluído entre 9 e 11 anos de estudo. As diferenças entre os serviços públicos e privados foram estatisticamente significativas para todas as variáveis sociodemográficas analisadas. Comparativamente às vítimas atendidas no setor privado, nas unidades públicas de saúde registrou-se maior proporção de pacientes com menos de 10 anos, de cor de pele preta/ parda e com menor número de anos de estudo. A proporção de pacientes idosos e com maior escolaridade foi $56 \%(R P=0,64)$ e 3,2 vezes $(R P$ $=0,31$ ) superior nos serviços de urgência e emer- gência privados em comparação aos públicos (Tabela 1).

Em relação às características do evento, descritas na Tabela 2, verificou-se que 35,8\% dos atendimentos registrados no município foram devidos a quedas e $15,9 \%$ a acidentes de transporte. As violências, compostas por lesões autoprovocadas, agressões e maus tratos, responderam juntas por $6,1 \%$ dos atendimentos. Foram observadas diferenças significativas entre os atendimentos nos serviços de urgência e emergência públicos/conveniados e os privados para todas as variáveis relativas às características do evento, com exceção das questões sobre consumo de bebida alcoólica pela vítima, embora a diferença quanto à suspeita de consumo de álcool tenha se aproximado do limiar de significância estatística. Os acidentes de transportes $(\mathrm{RP}=2,10)$, acidentes envolvendo animais $(\mathrm{RP}=2,77)$ e agressões e maus tratos $(\mathrm{RP}=2,13)$ foram cerca de 2 vezes mais frequentes nos serviços públicos de saúde do que nos privados. Os choques contra objeto/pessoa $(\mathrm{RP}=0,57)$ e entorses $(\mathrm{RP}=0,37)$ apresentaram maior proporção nos hospitais

Tabela 1. Características sociodemográficas dos pacientes atendidos por violências e acidentes, segundo natureza jurídica dos serviços de urgência e emergência ${ }^{1}$. VIVA - Campinas, 2009.

\begin{tabular}{|c|c|c|c|c|c|c|c|}
\hline \multirow{3}{*}{$\begin{array}{l}\text { Características } \\
\text { sociodemográficas }\end{array}$} & \multirow{2}{*}{\multicolumn{2}{|c|}{$\begin{array}{c}\text { Total } \\
(\mathrm{n}=1094) \\
\end{array}$}} & \multicolumn{4}{|c|}{ Natureza jurídica } & \multirow{3}{*}{$\begin{array}{c}\text { Razão } \\
\text { Público/Privado }\end{array}$} \\
\hline & & & \multicolumn{2}{|c|}{$\begin{array}{l}\text { Público } \\
(\mathrm{n}=742)\end{array}$} & \multicolumn{2}{|c|}{$\begin{array}{l}\text { Privado } \\
(\mathrm{n}=352)\end{array}$} & \\
\hline & n & $\%$ & n & $\%$ & n & $\%$ & \\
\hline Sexo & $0,012^{\star}$ & & & & & & \\
\hline Masculino & 653 & 59,7 & 462 & 62,3 & 191 & 54,3 & $1,15(0,97-1,36)$ \\
\hline Feminino & 441 & 40,3 & 280 & 37,7 & 161 & 45,7 & $0,82(0,68-1,00)$ \\
\hline Total & 1094 & & 742 & & 352 & & \\
\hline Faixa etária (anos) & $0,010^{*}$ & & & & & & \\
\hline 0 a 9 & 152 & 13,9 & 115 & 15,5 & 37 & 10,5 & $1,48(1,02-2,14)$ \\
\hline 10 a 19 & 200 & 18,3 & 139 & 18,8 & 61 & 17,3 & $1,08(0,80-1,46)$ \\
\hline 20 a 39 & 416 & 38,1 & 289 & 39,0 & 127 & 36,1 & $1,08(0,88-1,33)$ \\
\hline 40 a 59 & 208 & 19,0 & 131 & 17,7 & 77 & 21,9 & $0,81(0,61-1,07)$ \\
\hline 60 ou mais & 117 & 10,7 & 67 & 9,0 & 50 & 14,2 & $0,64(0,44-0,92)$ \\
\hline Total & 1093 & & 741 & & 352 & & \\
\hline Raça/ cor da pele & $0,000^{\star}$ & & & & & & \\
\hline Branca & 678 & 63,7 & 400 & 55,6 & 278 & 80,6 & $0,69(0,59-0,80)$ \\
\hline Preta/Parda & 375 & 35,3 & 310 & 43,1 & 65 & 18,8 & $2,29(1,75-2,99)$ \\
\hline Amarela/Indígena & 11 & 1,0 & 9 & 1,3 & 2 & 0,6 & $2,16(0,47-9,99)$ \\
\hline Total & 1064 & & 719 & & 345 & & \\
\hline Escolaridade (anos de estudo) & $0,000^{*}$ & & & & & & \\
\hline 0 a 4 & 227 & 24,1 & 176 & 28,1 & 51 & 16,3 & $1,72(1,26-2,35)$ \\
\hline 5 a 8 & 181 & 19,3 & 133 & 21,2 & 48 & 15,3 & $1,38(0,99-1,92)$ \\
\hline 9 a 11 & 359 & 38,2 & 251 & 40,0 & 108 & 34,5 & $1,16(0,92-1,45)$ \\
\hline 12 ou mais & 173 & 18,4 & 67 & 10,7 & 106 & 33,9 & $0,31(0,23-0,43)$ \\
\hline Total & 940 & & 627 & & 313 & & \\
\hline
\end{tabular}

${ }^{*}$ Valor de p do teste $\chi^{2} \cdot{ }^{1}$ Diferenças significativas ao nível de 5\% são indicadas em negrito. 
privados, sendo respectivamente $75 \%$ e 2,7 vezes superiores nestes em comparação aos públicos.

Os locais mais frequentes de ocorrência dos eventos foram a residência $(38,3 \%)$ e a via públi- ca $(28,4 \%)$. As ocorrências em via pública foram significativamente mais frequentes entre os pacientes atendidos nos serviços públicos ( $\mathrm{RP}=$ $1,70)$, enquanto as verificadas em locais de práti-

Tabela 2. Características dos eventos de violências e acidentes, segundo natureza jurídica dos serviços de urgência e emergência ${ }^{1}$. VIVA - Campinas, 2009.

\begin{tabular}{|c|c|c|c|c|c|c|c|}
\hline \multirow{3}{*}{ Características do evento } & \multirow{2}{*}{\multicolumn{2}{|c|}{$\begin{array}{c}\text { Total } \\
(\mathrm{n}=1094)\end{array}$}} & \multicolumn{4}{|c|}{ Natureza jurídica } & \multirow{3}{*}{$\begin{array}{c}\text { Razão } \\
\text { Público/Privado }\end{array}$} \\
\hline & & & \multicolumn{2}{|c|}{$\begin{array}{l}\text { Público } \\
(\mathrm{n}=742)\end{array}$} & \multicolumn{2}{|c|}{$\begin{array}{c}\text { Privado } \\
(\mathrm{n}=352)\end{array}$} & \\
\hline & $\mathbf{n}$ & $\%$ & $\mathbf{n}$ & $\%$ & $\mathbf{n}$ & $\%$ & \\
\hline Tipo de ocorrência & $0,000^{*}$ & & & & & & \\
\hline Acidente de transporte & 174 & 15,9 & 142 & 19,1 & 32 & 9,1 & $2,10(1,43-3,09)$ \\
\hline Queda & 392 & 35,8 & 263 & 35,4 & 129 & 36,6 & $0,97(0,78-1,19)$ \\
\hline Ferimento por objeto perfurocortante & 64 & 5,9 & 46 & 6,2 & 18 & 5,1 & $1,21(0,70-2,09)$ \\
\hline Acidente com animais & 41 & 3,8 & 35 & 4,7 & 6 & 1,7 & $2,77(1,16-6,58)$ \\
\hline Queda de objeto sobre pessoa & 44 & 4,0 & 35 & 4,7 & 9 & 2,6 & $1,84(0,89-3,84)$ \\
\hline Choque contra objeto/pessoa & 125 & 11,4 & 68 & 9,2 & 57 & 16,2 & $0,57(0,40-0,80)$ \\
\hline Entorses & 128 & 11,7 & 56 & 7,6 & 72 & 20,5 & $0,37(0,26-0,52)$ \\
\hline Outros acidentes & 59 & 5,4 & 44 & 5,9 & 15 & 4,3 & $1,39(0,77-2,50)$ \\
\hline Lesão autoprovocada & 12 & 1,1 & 8 & 1,1 & 4 & 1,1 & $0,95(0,28-3,15)$ \\
\hline Agressão/maus-tratos & 55 & 5,0 & 45 & 6,1 & 10 & 2,8 & $2,13(1,07-4,23)$ \\
\hline Total & 1094 & & 742 & & 352 & & \\
\hline Local de ocorrência & $0,000^{*}$ & & & & & & \\
\hline Residência & 411 & 38,3 & 260 & 35,9 & 151 & 43,4 & $0,83(0,68-1,01)$ \\
\hline Escola & 83 & 7,7 & 53 & 7,3 & 30 & 8,6 & $0,85(0,54-1,33)$ \\
\hline Local de prática esportiva & 96 & 9,0 & 40 & 5,5 & 56 & 16,1 & $0,34(0,23-0,51)$ \\
\hline Via pública & 304 & 28,4 & 237 & 32,8 & 67 & 19,3 & $1,70(1,30-2,23)$ \\
\hline Comércio/serviços/bar & 133 & 12,4 & 97 & 13,4 & 36 & 10,3 & $1,29(0,88-1,90)$ \\
\hline Outro & 45 & 4,2 & 37 & 5,1 & 8 & 2,3 & $2,22(1,03-4,77)$ \\
\hline Total & 1072 & & 724 & & 348 & & \\
\hline Natureza da lesão corporal & $0,000^{*}$ & & & & & & \\
\hline Sem lesão física & 62 & 5,9 & 30 & 4,2 & 32 & 9,3 & $0,45(0,28-0,75)$ \\
\hline Contusão & 278 & 26,4 & 197 & 27,8 & 81 & 23,6 & $1,18(0,91-1,53)$ \\
\hline Corte/laceração & 186 & 17,6 & 143 & 20,1 & 43 & 12,5 & $1,61(1,14-2,26)$ \\
\hline Entorse/luxação & 185 & 17,5 & 92 & 13,0 & 93 & 27,0 & $0,48(0,36-0,64)$ \\
\hline Fratura & 129 & 12,2 & 74 & 10,4 & 55 & 16,0 & $0,65(0,46-0,92)$ \\
\hline Traumatismo crânio-encefálico/politraumatismo & o 107 & 10,2 & 95 & 13,4 & 12 & 3,5 & $3,83(2,10-6,99)$ \\
\hline Outra & 107 & 10,2 & 79 & 11,1 & 28 & 8,1 & $1,37(0,89-2,10)$ \\
\hline Total & 1054 & & 710 & & 344 & & \\
\hline Parte do corpo atingida & $0,000^{*}$ & & & & & & \\
\hline Região cabeça/pescoço & 215 & 20,6 & 166 & 23,3 & 49 & 14,7 & $1,58(1,15-2,18)$ \\
\hline Coluna/tórax/abdome/quadril/genitais & 63 & 6,0 & 46 & 6,5 & 17 & 5,1 & $1,26(0,72-2,21)$ \\
\hline Membros superiores & 304 & 29,1 & 195 & 27,4 & 109 & 32,7 & $0,84(0,66-1,06)$ \\
\hline Membros inferiores & 382 & 36,6 & 236 & 33,1 & 146 & 43,9 & $0,76(0,61-0,93)$ \\
\hline Múltiplos órgãos/regiões & 81 & 7,7 & 69 & 9,7 & 12 & 3,6 & $2,69(1,46-4,96)$ \\
\hline Total & 1045 & & 712 & & 333 & & \\
\hline \multirow[t]{2}{*}{ Evento relacionado ao trabalho } & $0,002^{\star}$ & & & & & & \\
\hline & 225 & 21,0 & 172 & 23,7 & 53 & 15,4 & $1,54(1,13-2,09)$ \\
\hline \multirow[t]{2}{*}{ Suspeita de uso de bebida alcoólica } & $0,047^{\star}$ & & & & & & \\
\hline & 59 & 5,5 & 47 & 6,5 & 12 & 3,5 & $1,85(0,98-3,48)$ \\
\hline \multirow[t]{2}{*}{ Declaração de uso de bebida alcoólica } & $0,454^{*}$ & & & & & & \\
\hline & 75 & 7,1 & 54 & 7,4 & 21 & 6,2 & $1,20(0,73-1,99)$ \\
\hline
\end{tabular}

*Valor de p do teste $\chi^{2} .{ }^{1}$ Diferenças significativas ao nível de $5 \%$ são indicadas em negrito. 
ca esportiva foram 2,9 superiores nas vítimas atendidas nos hospitais privados $(\mathrm{RP}=0,34)$. Nas unidades públicas de saúde, também foi registrada maior proporção de atendimentos para os demais locais de ocorrência $(5,1 \%)$, os quais foram constituídos, neste setor, principalmente por indústria/construção $(56,8 \%)$ e habitação coletiva (13,5\%) (dados não apresentados) (Tabela 2).

As contusões foram a principal lesão corporal das vítimas de acidentes e violências tanto em unidades de saúde públicas ou conveniadas ao SUS $(27,8 \%)$ como nos hospitais privados $(23,6 \%)$, não apresentando diferença significativa entre os dois grupos. As frequências de atendimentos com cortes/laceração e traumatismo crânio-encefálico/politraumatismo foram, respectivamente, $61 \%$ e 3,8 vezes maiores nos serviços de saúde públicos ou conveniados ao SUS. Nos hospitais particulares, foram significativamente mais frequentes os acidentes e as violências sem lesão física $(R P=0,45)$, com entorses e luxações $(\mathrm{RP}=0,48)$ e com fraturas $(\mathrm{RP}=0,65)$. Lesões na região da cabeça e pescoço e em múltiplos orgãos e regiões foram $58 \%$ e 2,7 vezes mais frequentes nas unidades de saúde públicas em comparação ao registrado no setor privado. No entanto, em relação às lesões nos membros inferiores, os hospitais privados responderam pelas maiores proporções de atendimentos $(\mathrm{RP}=0,76)$. Os eventos que estavam relacionados ao trabalho apresentaram frequência significativamente maior nos serviços de urgência e emergência da rede pública/conveniada $(\mathrm{RP}=1,54)($ Tabela 2$)$.

O principal meio de locomoção para o paciente chegar à unidade de urgência e emergência foram os veículos particulares, com diferença significativa entre os atendimentos registrados no sistema público de saúde $(60,8 \%)$ e no particular $(82,6 \%)$ (Tabela 3). A frequência de transporte em ambulância, unidades de SAMU ou de resgate para os serviços públicos foi 2,7 vezes superior em relação aos particulares (14,3\% versus 5,3\%). A proporção de vítimas que usaram transporte coletivo para buscar atendimento foi 2 vezes maior nas unidades públicas e conveniadas ao SUS do que nas privadas. $\mathrm{O}$ uso de viaturas policiais por 15 vítimas, ainda que sem atingir o limiar de significância estatística, foi mais frequente entre os pacientes atendidos nos hospitais públicos do que nos privados. Das 14 vítimas transportadas pelas viaturas policiais ao setor público, 6 casos eram decorrentes de maus-tratos/agressões e 2 de lesões autoprovocadas (dados não apresentados).

A maioria dos eventos foi registrada nos períodos vespertino $(36,7 \%)$ e noturno $(31,5 \%)$, sem diferença significativa entre serviços públicos e privados. As proporções de atendimentos realizados aos domingos $(\mathrm{RP}=0,54)$ e sábados $(\mathrm{RP}=0,70)$ foram significativamente mais elevadas nos hospitais privados, enquanto as observadas nas segundas e quintas-feiras foram, respectivamente, $88 \%$ e $46 \%$ superiores no setor público. A maioria das vítimas recebeu alta após o atendimento na emergência tanto na rede pública $(85,4 \%)$ quanto na privada $(95,1 \%)$, sem que a diferença atingisse significância estatística. As vítimas que foram transferidas para serviços ambulatoriais totalizaram $8,3 \%$ dos atendimentos realizados no setor público contra apenas $0,6 \%$ no privado. Foram hospitalizados $4,8 \%$ dos pacientes atendidos nas unidades de saúde públicas e 4,0\% daqueles atendidos nos hospitais privados, sem diferença significativa entre os dois grupos (Tabela 3).

\section{Discussão}

Este estudo revelou importantes diferenças entre os atendimentos a vítimas de acidentes e violências realizados nos serviços de urgência e emergência públicos ou conveniados ao SUS e os privados no município de Campinas.

Cabe ressaltar que este recorte segundo a natureza jurídica do serviço de urgência e emergência não é frequente na literatura, tendo sido encontrados poucos estudos nacionais que se dedicaram a analisar diferenças entre unidades de saúde da rede pública ou conveniadas ao SUS e os da privada. Nesta escassa produção, as pesquisas têm privilegiado investigações sobre internações hospitalares ou mortalidade segundo a natureza jurídica do hospital ${ }^{10-13}$. Também, internacionalmente, estudos comparando atendimentos do setor público com os do setor privado não são frequentes ${ }^{14,15}$.

Verificou-se que o setor público ou conveniado do município responde por 67,8\% ((IC 95\% 65,0\%- 70,6\%) dos atendimentos à emergência provocados por causas externas. A elevada demanda aos serviços públicos de saúde do país é verificada por diferentes pesquisas. Estimativas nacionais baseadas nos dados dos Suplementos Saúde das PNAD (Pesquisa Nacional por Amostra de Domicílios) apontam que 56,7\% dos brasileiros que procuraram serviços de saúde nas duas semanas que antecederam o inquérito de 2008 relataram ter recebido atendimento na rede pública de saúde ${ }^{16}$. Ainda com base nos dados das PNAD, estudos identificam o SUS como o principal financiador das internações hospitala- 
Tabela 3. Características dos atendimentos por violências e acidentes, segundo natureza jurídica dos serviços de urgência e emergência ${ }^{1}$. VIVA - Campinas, 2009.

\begin{tabular}{|c|c|c|c|c|c|c|c|}
\hline \multirow{3}{*}{ Características do atendimento } & \multicolumn{6}{|c|}{ Natureza jurídica } & \multirow{3}{*}{$\begin{array}{c}\text { Razão } \\
\text { Público/Privado }\end{array}$} \\
\hline & \multicolumn{2}{|c|}{$\begin{array}{c}\text { Total } \\
(\mathrm{n}=1094)\end{array}$} & \multicolumn{2}{|c|}{$\begin{array}{l}\text { Público } \\
(\mathrm{n}=742)\end{array}$} & \multicolumn{2}{|c|}{$\begin{array}{l}\text { Privado } \\
(\mathbf{n}=352)\end{array}$} & \\
\hline & $\mathbf{n}$ & $\%$ & $\mathbf{n}$ & $\%$ & $\mathbf{n}$ & $\%$ & \\
\hline Meio de locomoção utilizado & $0,000^{*}$ & & & & & & \\
\hline A pé & 44 & 4,3 & 34 & 4,9 & 10 & 3,0 & $1,66(0,82-3,37)$ \\
\hline Veículo particular & 701 & 67,9 & 421 & 60,8 & 280 & 82,6 & $0,73(0,63-0,85)$ \\
\hline SAMU/Ambulância/Resgate & 117 & 11,3 & 99 & 14,3 & 18 & 5,3 & $2,69(1,63-4,44)$ \\
\hline Coletivo (Ônibus/Van) & 155 & 15,0 & 125 & 18,0 & 30 & 8,8 & $2,04(1,37-3,04)$ \\
\hline Viatura policial & 15 & 1,5 & 14 & 2,0 & 1 & 0,3 & $6,85(0,90-52,08)$ \\
\hline Total & 1032 & & 693 & & 338 & & \\
\hline Período de atendimento & $0,649^{*}$ & & & & & & \\
\hline Manhã (06:00-11:59) & 302 & 27,6 & 196 & 26,4 & 106 & 30,1 & $0,88(0,69-1,11)$ \\
\hline Tarde (12:00-17:59) & 401 & 36,7 & 276 & 37,2 & 125 & 35,5 & $1,05(0,85-1,29)$ \\
\hline Noite (18:00-23:59) & 345 & 31,5 & 238 & 32,1 & 107 & 30,4 & $1,05(0,84-1,32)$ \\
\hline Madrugada (24:00-05:59) & 46 & 4,2 & 32 & 4,3 & 14 & 4,0 & $1,08(0,58-2,03)$ \\
\hline Total & 1094 & & 742 & & 352 & & \\
\hline Dia de atendimento & $0,000^{*}$ & & & & & & \\
\hline Domingo & 105 & 9,6 & 56 & 7,5 & 49 & 13,9 & $0,54(0,37-0,80)$ \\
\hline Segunda & 119 & 10,9 & 95 & 12,8 & 24 & 6,8 & $1,88(1,20-2,94)$ \\
\hline Terça & 127 & 11,6 & 91 & 12,3 & 36 & 10,2 & $1,20(0,81-1,76)$ \\
\hline Quarta & 97 & 8,9 & 57 & 7,7 & 40 & 11,4 & $0,68(0,45-1,01)$ \\
\hline Quinta & 273 & 24,9 & 206 & 27,8 & 67 & 19,1 & $1,46(1,11-1,92)$ \\
\hline Sexta & 190 & 17,4 & 128 & 17,2 & 62 & 17,6 & $0,98(0,72-1,33)$ \\
\hline Sábado & 183 & 16,7 & 109 & 14,7 & 74 & 21,0 & $0,70(0,52-0,94)$ \\
\hline Total & 1094 & & 742 & & 352 & & \\
\hline $\begin{array}{l}\text { Evolução na emergência } \\
\text { (primeiras } 24 \text { horas) }\end{array}$ & $0,000^{*}$ & & & & & & \\
\hline Alta & 937 & 88,6 & 607 & 85,4 & 330 & 95,1 & $0,90(0,78-1,03)$ \\
\hline Internação hospitalar & 48 & 4,5 & 34 & 4,8 & 14 & 4,0 & $1,18(0,64-2,21)$ \\
\hline Encaminhamento ambulatorial & 61 & 5,8 & 59 & 8,3 & 2 & 0,6 & $14,40(3,52-58,92)$ \\
\hline Outro & 12 & 1,1 & 11 & 1,5 & 1 & 0,3 & $5,37(0,69-41,58)$ \\
\hline Total & 1058 & & 711 & & 347 & & \\
\hline
\end{tabular}

"Valor de $\mathrm{p}$ do teste $\chi^{2} .{ }^{1}$ Diferenças significativas ao nível de $5 \%$ são indicadas em negrito.

res $^{17}$, respondendo, em 2008, por $69,6 \%$ das hospitalizações do país ${ }^{18}$. Especificamente sobre internações hospitalares por causas externas nos serviços públicos e conveniados ao SUS, estimativas mostram um crescimento de $19,1 \%$ nas taxas ao longo da última década, bem como aumento na proporção de internações por causas acidentais ou violentas que passou de 7,7\% em 2000 para $10,4 \%$ em $2010^{19}$. Os dados da PNAD ainda revelam que a utilização de serviços do setor público ocorre também entre os portadores de planos e seguros privados $(13,4 \%)$ ou de planos de assistência ao servidor público $(13,8 \%)^{16}$. Em Campinas, cerca de 55\% da população possui algum plano de saúde privado.
O elevado volume de atendimentos de emergência na rede pública ou conveniada ao SUS de Campinas é resultante também da capacidade instalada deste setor para a atenção ao paciente vítima de violências e acidentes, contando com 5 unidades de pronto-atendimento e 3 hospitais de grande porte de referência ao trauma, com regulação médica de fluxo de paciente feita pelo SAMU.

O perfil sociodemográfico dos pacientes difere conforme a natureza jurídica das unidades de urgência e emergência, com maior proporção de utilização dos serviços do setor público pela população negra e parda e de baixa escolaridade, reforçando o que é constatado pela literatura 
sobre procura e uso de serviços de saúde ${ }^{16-18,20}$. Porto et al. ${ }^{18}$, baseado nos dados da PNAD, constataram que os atendimentos custeados pelo SUS no ano de 2008 foram preponderantes até o sétimo decil de renda familiar da população brasileira, a partir do qual os planos e seguros de saúde privados passam a responder massivamente pelo financiamento. Usando a PNAD 2003, Ribeiro et al. ${ }^{20}$ observaram que, no perfil dos usuários do SUS, predominava a cor/raça negra ou parda e baixa escolaridade. Desse modo, a maior proporção de utilização dos serviços de urgência e emergência do setor público pelos segmentos socioeconomicamente vulneráveis aponta que a qualidade dos serviços ofertados pelo SUS é fundamental na busca de redução das iniquidades em saúde, visto atender a população com as mais precárias condições de vida ${ }^{20}$.

Por outro lado, a maior proporção de atendimentos a idosos ocorrida nos hospitais privados resulta em grande parte da distinta composição etária dos segmentos sociais da população com e sem acesso aos planos de saúde privados, como tem sido registrado por estudos realizados com dados da PNAD ${ }^{21}$. Estima-se que 61\% na população com mais de 60 anos no município de Campinas é filiada a planos de saúde privados9. Vários estudos constataram um padrão mais envelhecido na cobertura por planos de assistência médica na saúde suplementar no país, com altos percentuais de beneficiários nas faixas etárias de idosos ${ }^{22}$ e especialmente vinculados a planos de saúde individuais ${ }^{21}$.

À semelhança do registrado no VIVA $2009^{7}$, as quedas despontam como a principal causa de atendimento em Campinas tanto no setor público quanto no privado. A seguir, destacam-se os acidentes de trânsito nas unidades públicas de saúde, assim como observado para os dados nacionais. No setor privado de Campinas, esta segunda posição é ocupada pelas entorses, ao passo que os acidentes de trânsito seriam a quarta causa mais frequente de atendimentos nestes serviços de saúde. Os choques contra objeto aparecem em terceiro lugar seja na esfera pública seja na privada. Todavia, ao englobar os dados nacionais de violências e acidentes num único ranking geral, os choques figurariam apenas na quinta posição, após as agressões/maus tratos e ferimentos por objeto perfurocortante.

Do exposto, torna-se evidente que não apenas há desigualdades nos padrões de distribuição dos tipos de ocorrências atendidos pelo setor privado e pelo público de Campinas, como também diferenças entre o perfil deste último e o indicado pelos dados nacionais que reúnem ape- nas atendimentos do SUS. Portanto, com exceção das similaridades nas duas principais causas de atendimento entre o setor público de Campinas e os dados nacionais, as variações nos rankings não devem ser unicamente atribuídas à inclusão do setor privado pelo inquérito de Campinas. Diferenças no perfil epidemiológico das ocorrências de eventos violentos e acidentais em Campinas em comparação ao delineado pelos dados globais das 23 capitais brasileiras e do Distrito Federal podem também explicar as variações encontradas na distribuição das causas.

O conjunto dos eventos violentos, formado por lesões autoprovocadas, maus tratos e agressões, representou 7,2\% nas ocorrências atendidas pelas unidades públicas de saúde de Campinas, enquanto estimativas nacionais do VIVA mostram valores mais elevados com 10,4\% em $2006^{8,23}$ e $10,1 \%$ em $2009^{7}$. Se comparadas estas estimativas nacionais do setor público com os dados levantados no setor privado de Campinas, a diferença se torna ainda maior, visto que os atendimentos decorrentes de violências responderam por apenas 3,9\% nos hospitais privados. Em Campinas, as agressões e maus tratos tiveram ocorrência 2 vezes superior nas unidades públicas em comparação nas privadas, o que está em consonância com a constatação de maior incidência dos distintos tipos de violência nos segmentos socialmente mais vulneráveis ${ }^{7,23,24}$.

O setor público foi a porta preferencial para as urgências e emergências de acidentes envolvendo animais, atendendo 3 vezes mais vítimas do que as unidades de saúde privadas. A maior procura por atendimento nas unidades públicas de saúde pode estar relacionada à existência de programas de vigilância e controle de zoonoses consolidados na rede pública, como de atendimento antirrábico. Outro fator de atração seria o Centro de Controle de Intoxicações (CCI) da UNICAMP, que é referência para o diagnóstico e o tratamento das exposições a animais peçonhentos.

Um importante achado deste estudo foi que alguns dados sinalizam maior gravidade na condição das vítimas conduzidas a hospitais públicos. Informações referentes à natureza da lesão corporal assinalam que traumatismos crânioencefálicos e politraumatismos apresentaram frequência significativamente superior nos hospitais públicos ou conveniados ao SUS, o que é consistente com o achado de maior proporção de casos com lesões na região da cabeça e pescoço e em múltiplos orgãos e regiões nestas unidades de saúde, embora o encaminhamento para internação hospitalar não tenha diferido entre os dois segmentos. Merece ainda ser destacado 
que, nos hospitais da rede privada, foi registrada maior proporção de casos que não resultaram em lesões físicas ou que tiveram entorses e luxações, as quais são consideradas lesões de menor gravidade. Estes achados indicariam maior consumo dos serviços públicos em situação de maior gravidade, o que poderia decorrer dos tipos de lesões que afetam com maior frequência os segmentos sociais SUS dependentes e também do maior encaminhamento dos casos graves, independentemente do nível socioeconômico da vítima, para as unidades públicas de referência para o trauma, que seguem as diretrizes de universalização do atendimento, acolhendo também os que possuem planos de saúde.

Vários estudos têm mostrado maior admissão de pacientes em condições mais graves no setor público ${ }^{11,12}$. Pesquisa, comparando índices de mortalidade hospitalar por causas naturais entre unidades de saúde públicas e privadas do Tennessee, Estados Unidos, revelou maior admissão de pacientes com quadros mais graves na rede pública em comparação à privada ${ }^{14}$. Martins et al. ${ }^{10}$, ao analisar indicadores de mortalidade hospitalar por doenças cardiovasculares e respiratórias na rede pública e privada no município de Ribeirão Preto, São Paulo, observaram que a esfera pública tratou de pacientes com quadros de maior gravidade, sugerindo que parte dos resultados poderiam ser explicados pelas características dos hospitais públicos com unidades de cuidado intensivo e pelas especialidades e habilidades do corpo clínico que trabalha nestas unidades. O estudo de Yazlle Rocha et al. ${ }^{13}$, também conduzido em Ribeirão Preto, mostrou diferenças no perfil de morbidade hospitalar segundo fonte de financiamento, com maior frequência de internações por lesões e envenamentos e de casos mais graves e complexos no SUS em comparação aos atendimentos realizados pelos serviços particulares ou via planos de saúde privados.

Ainda, tendo em vista a associação significativa entre atendimento pré-hospitalar e a gravidade das lesões ${ }^{25}$, a maior porcentagem de vítimas transportadas por ambulâncias, pelo SAMU ou unidades de resgate aos serviços públicos de saúde poderia sugerir que a rede pública é a principal responsável pelo atendimento de casos com lesões de maior gravidade. Todavia, cabe salientar que muitas vezes os serviços de resgate são utilizados com relativa independência em relação à gravidade ou complexidade do caso. Por outro lado, os pacientes que dispõem de condução própria mobilizam-se para os hospitais sem solicitar transporte aos serviços de atendimento préhospitalar ${ }^{25}$.
Embora sem diferença significativa entre os atendimentos realizados pelo setor público e pelo privado, é preciso mencionar que, das 15 ocorrências socorridas pelas viaturas policiais, $8 \mathrm{ca}$ sos eram de vítimas de violências, as quais foram todas transportadas para as unidades de urgência e emergência da rede pública. As estimativas nacionais do VIVA também apontam que as viaturas foram mais utilizadas no transporte de vítimas de violência $(10,1 \%)$ em comparação às de acidentes $(0,8 \%)^{7}$.

A presente pesquisa ainda detectou que o local onde ocorreu a grande maioria dos eventos acidentais e violentos em Campinas foi a residência, seguido da via pública tanto no setor público quanto no privado, diferenciando-se dos resultados de outros estudos do VIVA que mostram padrão inverso, com maior frequência nas vias públicas seja para as violências ${ }^{23,24}$, seja para os acidentes $^{26}$. Compete destacar ainda que, nos atendimentos realizados pelo setor público de Campinas, a proporção de eventos ocorridos em residência $(35,9 \%)$ foi próxima à registrada em via pública $(32,8 \%)$, mas no setor privado esses percentuais se distanciam bastante, sendo respectivamente de 43,4\% nos domicílios e 19,3\% nas vias públicas. A maior ocorrência de eventos nas residências somente foi verificada em pesquisas do VIVA específicas sobre quedas ${ }^{27}$ e aquelas com recortes para crianças menores de 10 anos $^{28}$. Em estudo sobre morbidade hospitalar em unidades de referência no município de São Paulo, Gawryszewski et al. ${ }^{29}$ também identificaram a residência como o local de maior frequência entre as quedas, lesões autoprovocadas e queimaduras.

A maior proporção de eventos $(23,7 \%)$ relacionados ao trabalho atendidos nos serviços públicos de saúde de Campinas, em comparação a outros trabalhos com dados do VIVA ${ }^{27}$, sinaliza questão que merece posterior investigação. Como registrado neste presente estudo, também outras pesquisas sobre acidentes de trabalho indicaram maior proporção de atendimentos na rede pública de saúde ${ }^{30,31}$. Em estudo conduzido em Salvador, Bahia, foi verificado que $71,0 \%$ de todos os atendimentos foram financiados pelo SUS independentemente do nível socioeconômico do trabalhador, embora com variações significativas quanto à formalidade do contrato de trabalho, indicando que a rede pública de saúde assume grande responsabilidade no atendimento de acidentes e violências que afetam os trabalhadores ${ }^{31}$.

Em relação à evolução nas primeiras 24 horas, verificou-se que, com exceção do encaminhamento ambulatorial, não houve diferença significativa entre o setor público e o privado. No en- 
tanto, em pesquisa conduzida em serviços médicos de emergência em Kentucky, nos Estados Unidos, constatou-se maior proporção de internações hospitalares entre indivíduos com planos de saúde privado em comparação aos sem seguro saúde e aqueles cobertos pelos planos públicos, após ter controlado pela gravidade das lesões ${ }^{32}$.

O conjunto dos achados deste estudo deve considerar a presença de algumas limitações. $\mathrm{O}$ perfil epidemiológico dos atendimentos realizados nas unidades públicas, obtido por este estudo, é representativo de Campinas, uma vez que a amostra abarcou a totalidade dos oito serviços públicos ou conveniados ao SUS do município. Quanto ao setor privado, a amostra não abrangeu todos os serviços existentes no município, embora tenha incluído os mais significativos, que respondem por aproxidamente $90 \%$ dos atendimentos a emergências desse setor. Outra limitação do estudo refere-se ao fato de que, embora o número de entrevistas conduzidas tenha superado o tamanho mínimo definido para a amostra, ele foi insuficiente para detectar possíveis diferenças em eventos menos frequentes. As análises deste estudo referem-se a todos os atendimentos realizados e não apenas aos destinados aos moradores de Campinas, embora estes representem a maioria, visto que apenas $11,6 \%$ dos pacientes não residiam no município.

A inclusão de unidades de saúde da rede privada na amostra do VIVA Campinas permitiu análise inédita dentro deste inquérito, ao viabilizar a comparação dos perfis epidemiológicos das vítimas de acidentes e violências atendidas em serviços de urgência e emergência públicos ou conveniados ao SUS e nos privados. Possibilitou melhor compreensão das características dos eventos violentos e acidentais e identificação das diferenças significativas entre os padrões de atendimentos realizados pela rede pública e privada do município.

Em síntese, a pesquisa mostrou que o setor público é o principal financiador dos serviços utilizados pelas vítimas de violências e acidentes, respondendo por quase $70 \%$ dos atendimentos. Em comparação ao setor privado, as unidades de saúde pública registraram maior proporção de vítimas de violências e acidentes com menos de 10 anos, cor de pele preta/parda e menor número de anos de estudo. Também foi verificada na rede pública maior frequência para: atendimentos por acidentes de trânsito, acidentes com animais e agressões/maus tratos; ocorrências em vias públicas; eventos relacionados ao trabalho; vítimas encaminhadas pelos serviços do SAMU/ ambulância/resgate; pacientes utilizando transportes coletivos; atendimentos ocorridos nas segundas e quintas-feiras; e pacientes direcionados para tratamento ambulatorial. Os dados ainda sugerem que os casos mais graves, com cortes/ lacerações e traumatismos crânio-encefálicos/ politraumatismos e com lesões na cabeça/pescoço e em múltiplos órgãos/regiões, são atendidos majoritariamente pelos serviços públicos e conveniados ao SUS. Esta maior proporção de atendimentos a casos potencialmente mais graves no sistema público de saúde pode ser um indicativo da oferta de recursos especializados e/ou da qualificação da equipe médica para o cuidado a vítimas de violências e acidentes com grande atração para os hospitais de referência para o trauma no município.

Os resultados do estudo apontam a relevância da inclusão periódica de unidades assistenciais do setor privado nos inquéritos VIVA de forma a viabilizar acompanhamento das diferenças do perfil epidemiológico dos acidentes e violências entre os dois setores. Esta iniciativa contribui com subsídios relevantes para decisões políticas na área de urgência e emergência tanto no planejamento de estratégias quanto na avaliação dos serviços, possibilitando definir com maior especificidade as ações da rede SUS e não SUS.

\section{Colaboradores}

AP Belon realizou a proposta do estudo, revisão da literatura, análise e interpretação dos dados e redação do artigo. NYJ Silveira coordenou o trabalho de campo do inquérito, participou da análise e interpretação dos dados e contribuiu na revisão crítica do artigo. MBA Barros elaborou a proposta do estudo, orientou a análise e interpretação dos dados e contribuiu na revisão crítica do artigo. C Baldo foi responsável pelas análises estatísticas. MMA Silva contribuiu na revisão crítica do artigo. Todos os autores aprovaram a versão final do artigo. 


\section{Agradecimentos}

À Organização Pan-Americana de Saúde pelo suporte financeiro concedido ao Centro Colaborador em Análise de Situação de Saúde da Faculdade de Ciências Médicas da Universidade Estadual de Campinas para análise dos dados. Ao Conselho Nacional de Desenvolvimento Científico e Tecnológico $(\mathrm{CNPq})$ pela bolsa de produtividade de MBA Barros.

A todas as unidades de saúde da rede SUS (Hospital Municipal Dr. Mário Gatti, Complexo Hospitalar Ouro Verde, Hospital de Maternidade Celso Pierro-PUCCAMP, Hospital de Clínicas da UNICAMP, Pronto Atendimento Padre Anchieta, Pronto Atendimento Centro, Pronto Atendimento Sérgio Arouca, Pronto Atendimento São José) e não SUS (Casa de Saúde, Centro Médico, Hospital Vera Cruz, Hospital Samaritano, Hospital Metropolitano, Real Sociedade Portuguesa de Beneficência, Hospital Irmãos Penteado) por terem aceitado o convite para participar do inquérito. A todos os entrevistadores e supervisores pelos esforços em coletar os dados com fidedignidade.

\section{Referências}

1. Reichenheim ME, Souza ER, Moraes CL, Jorge MHPM, Silva CMFP, Minayo MCS. Violence and injuries in Brazil: the effect, progress made, and challenges ahead. Lancet 2011; 6736(11):75-89.

2. Pan American Health Organization (PAHO). Información y análisis de salud: situación de salud en las Américas: indicadores básicos 2009. Washington, DC: PAHO; 2009.

3. Mascarenhas MDM, Monteiro RA, Sá NNB, Gonzaga LAA, Neves ACM, Roza DL, Silva MMA, Duarte EC, Malta DC. Epidemiologia das causas externas no Brasil: mortalidade por acidentes e violências no período de 2000 a 2009. In: Brasil. Ministério da Saúde (MS). Secretaria de Vigilância em Saúde. Departamento de Análise de Situação em Saúde. Saúde Brasil 2010: uma análise da situação de saúde e de evidências selecionadas de impacto de ações de vigilância em saúde. Brasília: MS; 2011. p. 225-249.

4. Belon AP, Barros MBA. Esperança de vida ao nascer: impacto das variações na mortalidade por idade e causas de morte no Município de Campinas, São Paulo, Brasil. Cad Saude Publica 2011; 27(5):877-887.

5. Barros MBA, Marín-León L, Almeida SDM, Restitutti MC, Belon AP. Mortalidade por causas externas. Boletim de Mortalidade n. 39. Campinas: s.n., 2007 [acessado 2012 mar 23]. Disponível em: http:/ /www.fcm.unicamp.br/centros/ccas/index.php? option=com_content\&view=article\&id=90\&Itemid=85

6. Marín-León L, Belon AP, Barros MBA, Almeida SDM, Restitutti MC. Tendência dos acidentes de trânsito em Campinas, São Paulo, Brasil: importância crescente dos motociclistas. Cad Saude Publica 2012; 28(1):39-51.

7. Brasil. Ministério da Saúde (MS). Secretaria de Vigilância em Saúde. Departamento de Análise de Situação de Saúde. Viva: vigilância de violências e acidentes, 2008 e 2009. Brasília: MS; 2010.

8. Brasil. Ministério da Saúde (MS). Secretaria de Vigilância em Saúde. Departamento de Análise de Situação de Saúde. Viva: vigilância de violências e acidentes, 2006 e 2007. Brasília: MS; 2009.

9. Brasil. Ministério da Saúde. Agência Nacional de Saúde Suplementar. ANS TABNET. [acessado em 2012 mar 23]. Disponível em: http://www.ans.gov.br/anstabnet/anstabnet/deftohtm.exe?anstabnet/dados/ TABNET_02.DEF.

10. Martins M, Blais R, Leite IC. Mortalidade hospitalar e tempo de permanência: comparação entre hospitais públicos e privados na região de Ribeirão Preto, São Paulo, Brasil. Cad Saude Publica 2004; 20(Supl. 2):S268-S282.

11. Iucif Jr N, Rocha JSY. Sistema de informações hospitalares como ajuste de risco em índices de desempenho. Rev Saude Publica 2004; 38(6):780-786.

12. Yazlle Rocha JS, Simões BJG. Estudo da assistência hospitalar pública e privada em bases populacionais, 1986-1996. Rev Saude Publica 1999; 33(1):44-54.

13. Yazlle Rocha JS, Simoes BJG, Guedes GLM. Assistência hospitalar como indicador da desigualdade social. Rev Saude Publica 1997; 31(5):479-487. 
14. Burns R, Nichols LO, Graney MJ, Applegate WB. Mortality in a Public and a Private Hospital compared: The Severity of Antecedent Disorders in Medicare Patients. Am J Public Health 1993; 83(7):966-971.

15. Montagu DD, Anglemyer A, Tiwari M, Drasser K, Rutherford GW, Horvath T, Kennedy GE, Bero L, Shah N, Kinlaw HS. Private versus public strategies for health service provision for improving health outcomes in resource-limited settings. San Francisco, CA: Global Health Sciences, University of California, San Francisco; 2011.

16. Silva ZP, Ribeiro MCSA, Barata RB, Almeida MF. Perfil sociodemográfico e padrão de utilização dos serviços de saúde do Sistema Único de Saúde (SUS), 2003-2008. Cien Saude Colet 2011; 16(9):3807-3816.

17. Porto SM, Santos IS, Ugá MAD. A utilização de serviços de saúde por sistema de financiamento. Cien Saude Colet 2006; 11(4):895-910.

18. Porto SM, Ugá MAD, Moreira RS. Uma análise da utilização de serviços de saúde por sistema financeiro: Brasil, 1998-2008. Cien Saude Colet 2011; 16(9):3795-3806.

19. Mascarenhas MDM, Monteiro RA, Sá NNB, Gonzaga LAA, Neves ACM, Silva MMA, Malta DC. Epidemiologia das causas externas no Brasil: morbidade por acidentes e violências. In: Brasil. Ministério da Saúde (MS). Secretaria de Vigilância em Saúde. Departamento de Análise de Situação em Saúde. Saúde Brasil 2010: uma análise da situação de saúde e de evidências selecionadas de impacto de ações de vigilância em saúde. Brasília: MS; 2011. p. 203-224.

20. Ribeiro MCSA, Barata RB, Almeida MF, Silva ZP. Perfil sociodemográfico e padrão de utilização de serviços de saúde para usuários e não-usuários do SUS - PNAD 2003. Cien Saude Colet 2006; 11(4):10111022.

21. Bahia L, Ronir RL, Salm C, Coata AJL, Kale PL Cavalcanti ML. O mercado de planos e seguros de saúde no Brasil: uma abordagem exploratória sobre a estratificação das demandas segundo a PNAD 2003. Cien Saude Colet 2006; 11(4):951-965.

22. Veras RP, Caldas CP, Araújo DV, Kuschnir R, Mendes W. Características demográficas dos idosos vinculados ao sistema suplementar de saúde no Brasil. Rev Saude Publica 2008; 42(3):497-502.

23. Mascarenhas MDM, Silva MMA, Malta DC, Moura L, Macário EM, Gawryszewski VP, Morais Neto OL. Perfil epidemiológico dos atendimentos de emergência por violência no Sistema de Serviços Sentinelas de Vigilância de Violências e Acidentes (VIVA) Brasil, 2006. Epidemiol Serv Saude 2009; 18(1):17-28.
24. Gawryszewski VP, Silva MMA, Malta DC, Kegler SR, Mercy JA, Mascarenhas MDM, Morais Neto OL. Violence-related injury in emergency departments in Brazil. Pan Am J Public Health 2008; 24(6): 400-408.

25. Ladeira RM, Barreto SM. Fatores associados ao uso de serviço de atenção pré-hospitalar por vítimas de acidentes de trânsito. Cad Saude Publica 2008; 24(2):287-294.

26. Mascarenhas MDM, Silva MMA, Malta DC, Moura L, Gawryszewski VP, Costa VC, Souza MFM, Morais Neto OL. Atendimentos de emergência por acidentes na Rede de Vigilância de Violências e Acidentes - Brasil, 2006. Cien Saude Colet 2009; 14(5):1657-1668.

27. Malta DM, Silva MMA, Mascarenhas MDM, Sá NNB, Morais Neto OL, Bernal RTI, Monteiro RA, Andrade SSCA, Gawryszewski VP. Características e fatores associados às quedas atendidas em serviços de emergência. Rev Saude Publica 2012; 46(1):128-137.

28. Malta DC, Mascarenhas MDM, Silva MMA, Macario EM. Perfil dos atendimentos de emergência por acidentes envolvendo crianças menores de dez anos: Brasil, 2006 a 2007. Cien Saude Colet 2009; 14(5):16691679.

29. Gawryszewski VP, Scarpelini S, Dib JA, Jorge MHPM, Pereira Junior GA, Morita M. Atendimentos de emergência por lesões decorrentes de causas externas: características das vítimas e local de ocorrência, Estado de São Paulo, Brasil, 2005. Cad Saude Publica 2008; 24(5):1121-1129.

30. Cordeiro R, Sakate M, Clemente APG, Diniz CS, Donalisio MR. Subnotificação de acidentes do trabalho não fatais em Botucatu, SP, 2002. Rev Saude Publica 2005; 39(2):254-260.

31. Santana VS, Araújo GR, Espírito-Santo JS, AraújoFilho JB, Iriart J. A utilização de serviços de saúde por acidentados de trabalho. Rev Bras Saude Ocup 2007; 32(115):135-143.

32. Svenson JE, Spurlock CW. Insurance Status and Admission to Hospital for Head Injuries: Are We Part of a Two-Tiered Medical System? Am J Emerg Med 2001; 19(1):19-24.

Artigo apresentado em 10/06/2012

Aprovado em 04/07/2012

Versão final apresentada em 14/07/2012 